\title{
Measuring the Force of Interaction between a Metallic Probe and a Single Molecule
}

\author{
B. Naydenov, P. Ryan, L. C. Teague, ${ }^{*}$ and John J. Boland ${ }^{\dagger}$ \\ ${ }^{1}$ School of Chemistry and the Centre for Research on Adaptive Nanostructures and Nanodevices (CRANN), Trinity College, \\ Dublin 2, Ireland \\ (Received 8 February 2006; published 1 September 2006)
}

\begin{abstract}
Precision current measurements are recorded at $5 \mathrm{~K}$ during the approach and contact between a Pt-inked probe and the carbon-carbon double-bond region of an isolated 1,3-cyclohexadiene molecule chemisorbed on a $\mathrm{Si}(100)$ surface. Scanning tunneling spectroscopic data reveal systematic features in the current at specific probe-molecule separations. Aided by density functional theory calculations, we show that these features arise from interaction forces between the probe and molecule, which can be interpreted as the relaxation of the probe-molecule system prior to and during contact.
\end{abstract}

DOI: 10.1103/PhysRevLett.97.098304

There is enormous interest in the potential use of molecules in future electronic device technologies [1-3]. Although molecular electronics has the potential for the highest possible integration densities, the major difficulty in advancing this technology is establishing reliable contacts with molecules $[1,4]$. Not only is the influence of the contacting metal not well understood but the manner in which the contact perturbs the properties of molecules is a complete unknown [2,5], and precludes the rational design of molecular devices. In this Letter we introduce a novel STM-based method to measure the contact forces and interactions between a metallic probe and a single molecule. We show that, for a Pt-metal- "inked" STM probe and a 1,3-cyclohexadiene (1,3-CHD) molecule on the Si(100) surface, there is a repulsive barrier at large separations followed by an attractive interaction associated with contact and chemical bond formation. This method is quite general and applicable to a wide range of molecular systems.

To study the interaction between a single molecule and a STM probe it is necessary to develop a reliable method to control the chemical composition of the probe. This is accomplished by placing a single crystal metal sample [in this case $\mathrm{Pt}(111)]$ and the $\mathrm{Si}(100)$ substrate $\mathrm{s}[n+$ -type As, $<5 \mathrm{~m} \Omega \cdot \mathrm{cm}]$ together in a sample holder that allows each to be heated and prepared. Each sample was then characterized by LEED, exposed at room temperature to less than $0.1 \%$ of a monolayer of $1,3-\mathrm{CHD}$, cooled to $80 \mathrm{~K}$, transferred into a Createc LT-STM and cooled to $5 \mathrm{~K}$ for imaging [see Figs. 1(a) and 1(b)] and spectroscopic analysis [see Figs. 1(c)-1(e)]. Tungsten STM probes were cleaned by electron bombardment and then inked by drawing Pt atoms from the surface onto the end of the probe (see below). Figure 1(c) shows that the current increases exponentially during the approach to the $\operatorname{Pt}(111)$ surface, after which there is a sudden jump associated with contact formation [6-8]. The exponential behavior of the current $I$ follows the well-known distance dependence of the tunneling current [9-11]:

$$
I \sim \exp \left(-A \phi_{A}^{1 / 2} Z\right)
$$

where $\phi_{A}$ is the apparent tunneling barrier height, $Z$ is the
PACS numbers: 82.37.Gk, 39.25.+k, 68.43.-h, 81.16.Ta

probe-surface separation, and $A=2 \pi\left(8 m_{e}\right)^{1 / 2} / h$. The Pt surface is locally melted in the contact area and when the probe is withdrawn there is a clear hysteresis in the current due to the formation of a Pt metal neck that extends between the probe and the Pt surface [12] but which eventually breaks as the probe pulls away [see Fig. 1(c)]. The process can be repeated to insure that the probe is thoroughly inked with Pt atoms and after which measurements of $\phi_{A}$ using Eq. (1) yield a value of $5.5 \mathrm{eV}$, consistent with a Pt-Pt tunneling junction [13] (see below).
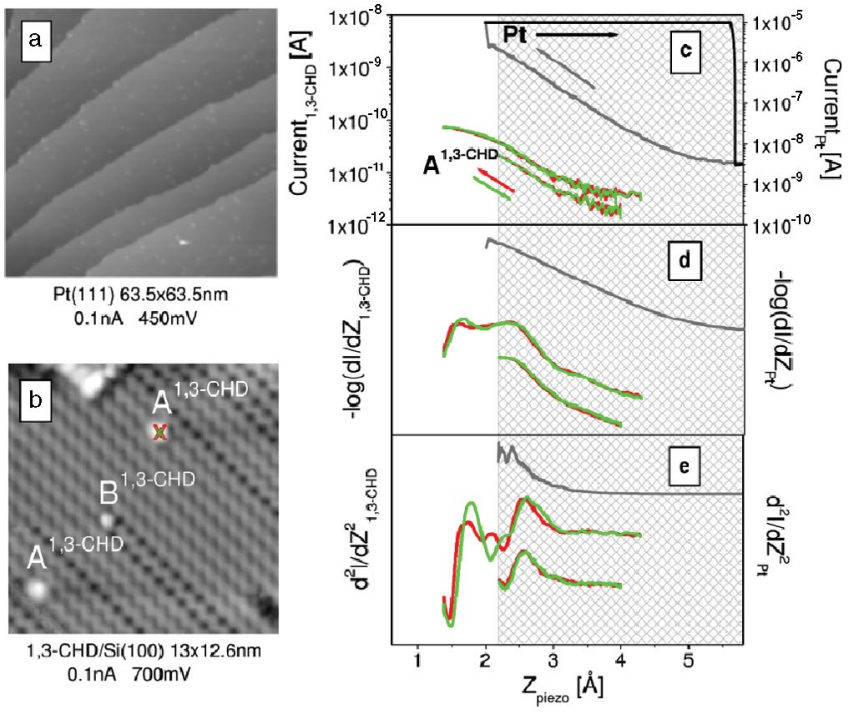

FIG. 1 (color). STM images of (a) $63.5 \times 63.5 \mathrm{~nm} \mathrm{Pt}(111)$ surface area with $0.1 \mathrm{nA}$ and $0.45 \mathrm{~V}$ sample bias and

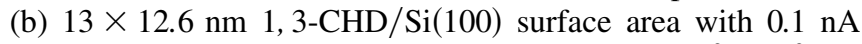
and $0.7 \mathrm{~V}$ sample bias. (c) $I$, (d) $d I / d Z$, and (e) $d^{2} I / d Z^{2}$ are simultaneously recorded on the bare $\mathrm{Pt}(111)$; gray (inwards to surface) and black (outwards), and on the 1,3-CHD maximum red (inwards) and green (outwards). The bottom curves in (c)(e) are from a separate 1,3-CHD experiment and are shifted with respect to the 1,3-CHD-ordinate scale to allow comparison. The spectra were taken with constant rate of $0.32 \AA / \mathrm{s}$. The crosshatched area in (c)-(e) indicates the hysteresis-free range of probe-sample separations. 
After the probe tip has been characterized in this manner, it was withdrawn for the Pt surface, laterally translated, positioned above the $\mathrm{Si}(100)$ surface and then brought carefully into tunneling contact. Figure 1(b) shows that at $5 \mathrm{~K}$ the $\mathrm{Si}(100)$ buckled dimers form weakly ordered $c(4 \times 2)$ and $p(2 \times 2)$ domains [14], in addition to 1,3CHD molecules bound to the surface in two different bonding configurations [15]. The STM probe tip was positioned over the maximum of the bright feature [ labeled A in Fig. 1(b)], which is known to be associated with the $\mathrm{C}=\mathrm{C}$ bond region of the 1,3-CHD molecule in the intradimer [2 + 2] configuration [15], and the approach experiment was repeated at a sample bias of $200 \mathrm{meV}$. The low bias was chosen because within the corresponding tunneling energy window the local density of states (LDOS) was measured to be independent of the probe-molecule separation [16]. Experimentally, the approach involved applying a $0.01 \mathrm{~nm}$ dither at $534 \mathrm{~Hz}$ to the $Z$ piezo under openloop conditions so that the current and the first and second harmonics $\left(d I / d Z\right.$ and $\left.d^{2} I / d Z^{2}\right)$ are measured simultaneously using a dual-phase lock-in amplifier (SR/7265), and shown in Figs. 1(c)-1(e). In contrast to the Pt case [gray curve in Fig. 1(c)], the current does not increase as a simple exponential during the approach nor is there a dramatic hysteresis between the inward (red) and outward (green) traces. Instead, the current shows systematic increases and decreases in the rate of current increase that is most clearly evident from the first and second harmonic measurements [Figs. 1(d) and 1(e)]. The current is essentially hysteresis-free provided the extent of the approach is confined to the crosshatched region in Figs. 1(c)-1(e) (bottom curves). The deviations between the traces observed at small separations, beyond the hysteresis-free region are attributed to weak-bond making and breaking in the probe-1,3-CHD-Si dimer complex.

To gain additional insight into the behavior of the current during the approach the tunneling barrier height was determined $[8,10]$ using the data in Figs. 1(c)-1(e). The simultaneous measure of the current $I$ and its first and second harmonics, provide three nominally equivalent methods to determine the barrier, which are shown in Figs. 2(a)-2(c). Namely,

$$
\begin{gathered}
\phi_{A 1}=[-d I / d Z / I A]^{2}, \quad \phi_{A 2}=d^{2} I / d Z^{2} / I A^{2}, \\
\phi_{A 3}=\left[-d^{2} I / d Z^{2} /(d I / d Z A)\right]^{2} .
\end{gathered}
$$

In the case of the Pt surface [gray curves in Figs. 2(a)-2(c)] the same dependence of the barrier on separation is found regardless of the method used $\left(\phi_{A 1}=\phi_{A 2}=\phi_{A 3}=\phi_{A}\right)$. Initially, during the approach, the barrier is approximately constant at $5.5 \mathrm{eV}$ followed by a small monotonic increase until an abrupt jump into contact, in agreement with the theory of metal-metal point contacts [6,7]. However, the barrier determined when the 1,3-CHD molecule is approached [red curves in Figs. 2(a)-2(c)] is not only dependent on the method used but each shows reproducible features at specific probe-molecule separations. In all

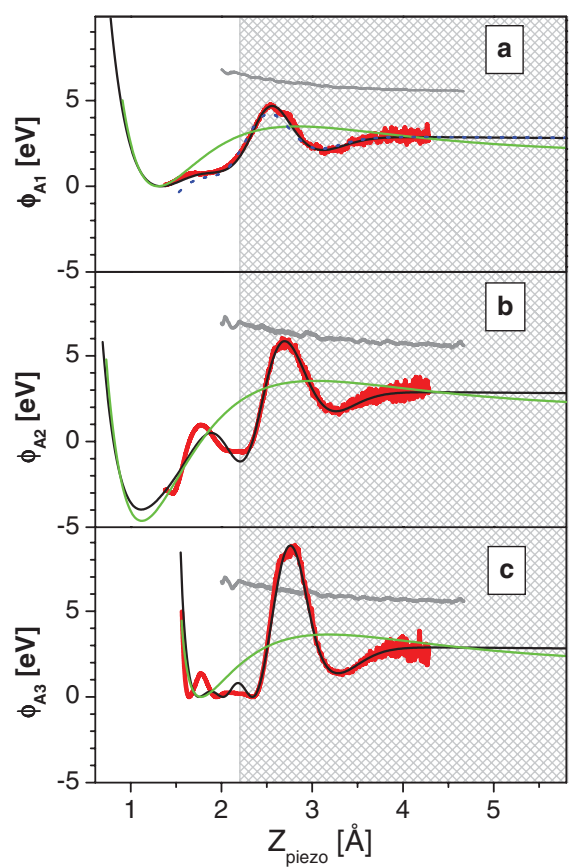

FIG. 2 (color). The dependence of the apparent tunneling barrier height $\phi_{A}$ on the probe-sample separation $Z_{\text {piezo }}$. The gray and red curves represent experimental results on bare $\operatorname{Pt}(111)$ surface, and on the 1,3-CHD molecule, respectively, derived from Eq. (2). The molecule data (red curves) are averages of the inward and outward traces in Fig. 1. The black and green curves are fits to these experimental data using Eq. (4), with (black) and without (green) a barrier interaction term in the potential. The crosshatched area indicates the hysteresis-free range in Figs. 1(c)-1(e). For comparison, the force gradient $(d F / d Z)$ derived from the black curve is presented as a dotted blue curve. The $d F / d Z$ amplitude is rescaled and vertically shifted to emphasize the qualitative agreement with the red curve in (a).

cases, the apparent barrier decreases slowly as the probe approaches, but then shows a pronounced maximum, followed by a secondary maximum at smaller separations, where the barrier approaches zero, or becomes negative in the case of the $\phi_{A 2}$ method.

To better understand this behavior we performed DFT calculations involving a 1,3-CHD molecule attached to a $\mathrm{Si}(100)$ dimer and above which a Pt probe [modeled as a five atom (100) cluster] was positioned over the $\mathrm{C}=\mathrm{C}$ of the molecule. The structure of the tip was constrained to the bulk. The Si(100) surface was modeled as a three-dimer long $3 \times 2$ slab, six layers deep and terminated by a $\mathrm{H}$ atom on the lower surface. Calculations were performed using the plane wave (cutoff energy $240 \mathrm{eV}$ ) and the PW91 GGA methods [17] with a $1 \times 3 \times 2$ Monkhorst-Pack grid [18], for which the energy and maximum force convergences were set to $2 \times 10^{-5} \mathrm{eV} /$ atom, and $0.05 \mathrm{eV} / \AA$, respectively [19]. With the probe located $2 \mathrm{~nm}$ away and the bottom two Si layers constrained to the bulk, the molecule-surface system was allowed to relax to determine the total energy of the isolated systems. All Si atoms were then fixed at their optimized positions and the molecule 
coordinates were recorded as a function of the separation between the probe and the surface.

Assuming a simplified model sketched in Figs. 3(b) and 3(c) in which the $\mathrm{C}=\mathrm{C}$ moiety is attached by a spring $\left(k_{S}\right)$ to the surface and responds to a force $F_{P}$ exerted on it by the probe. The calculated $\mathrm{C}=\mathrm{C}$ coordinates were used to determine the vertical displacement $\Delta Z_{\mathrm{C}=\mathrm{C}}$, and so the restoring force on the spring is $-F_{S}=k_{S} \Delta Z_{\mathrm{C}=\mathrm{C}}$, which in equilibrium $\left(-F_{S}=F_{P}\right)$ defines the force $\left(F_{P}\right)$ between the $\mathrm{C}=\mathrm{C}$ moiety and the probe. The interaction potential derived from this model assuming a spring constant $k_{s}$ of $20 \mathrm{~N} / \mathrm{m}$ (see below) is shown as solid black curve in Fig. 4. Regardless of the choice of spring constant there is an initial weak attractive interaction between the probe and molecule and at a distance of about $4 \AA$ there is a barrier (see inset in Fig. 4), followed by an attractive bonding interaction. A detailed analysis reveals that this barrier is associated with rehybridization due to a weak $p \pi \rightarrow d \pi$ bonding interaction in which charge is transferred from the $\mathrm{C}=\mathrm{C}$ bond to an empty $d$ orbital on the apex atom on the probe [20]. Prompted by these computational results, we directly account for this interaction force $F=\left|F_{P}\right|=\left|F_{S}\right|$ by writing the change in the actual separation $Z$ between the probe and the molecules as

$$
Z=Z_{\text {piezo }}-\Delta Z_{\mathrm{C}=\mathrm{C}}=Z_{\text {piezo }}+k_{S}^{-1} F(Z)
$$

where $Z_{\text {piezo }}$ is the tunneling junction width driven by the piezoscanner motion and $k_{S}$ is a fixed spring constant of the $\mathrm{C}=\mathrm{C}$ moiety attachment to the surface, with an equilib-

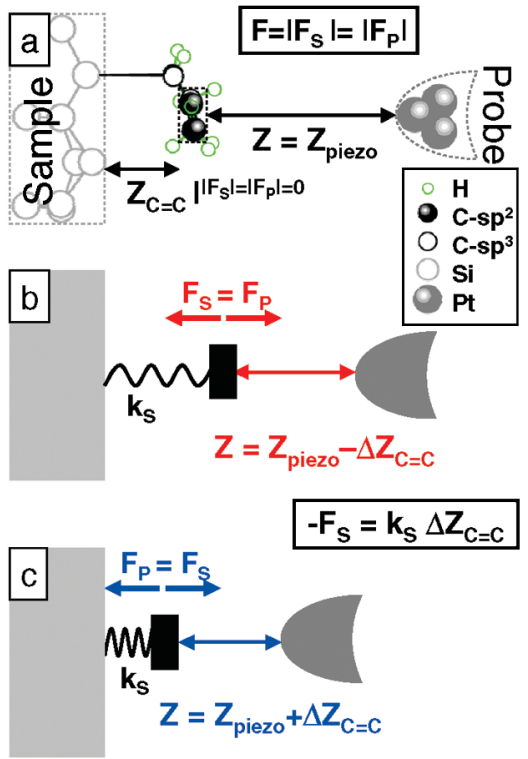

FIG. 3 (color). Schematic model of the probe-sample system under investigation. The $\mathrm{C}=\mathrm{C}$ moiety attachment to the surface is modeled by a spring that responds to the presence of the approaching probe. The parameters applied in the fitting procedure are illustrated in the figure. The different panels represent the model with no probe-molecule interaction (a), and with attractive (b) and repulsive (c) interaction. rium distance $Z_{\mathrm{C}=\mathrm{C}}$ when $F=0$. In this model (Fig. 3), when $F_{P}(Z)$ is attractive, a forward displacement of the piezo combines to produce a larger effective reduction in the gap between the $\mathrm{C}=\mathrm{C}$ and the probe, so that the current will increase faster than predicted by Eq. (1). Thus the deviations of the current and its harmonics from the exponential behavior in Eq. (1) reflect the motion and relaxation of the $\mathrm{C}=\mathrm{C}$ moiety during the approach.

We now use Eq. (3) to account for the influence of these forces and rewrite Eq. (2) as

$$
\begin{aligned}
\phi_{A 1}= & \phi_{A}\left(1+k_{S}^{-1} d F / d Z\right)^{2}, \\
\phi_{A 2}= & \phi_{A}\left[\left(1+k_{S}^{-1} d F / d Z\right)^{2}+\left(A \phi_{A}^{1 / 2}\right)^{-1} k_{S}^{-1} d^{2} F / d Z^{2}\right], \\
\phi_{A 3}= & \phi_{A}\left[\left(1+k_{S}^{-1} d F / d Z\right)^{2}+\left(A \phi_{A}^{1 / 2}\right)^{-1} k_{S}^{-1} d^{2} F / d Z^{2}\right](1 \\
& \left.+k_{S}^{-1} d F / d Z\right)^{-1} .
\end{aligned}
$$

Thus a nonlinear $F(Z)$ will produce additional terms in Eq. (4) apart from $\phi_{A}$ and will introduce nonlinearities into the dependence of $\phi_{A 1-3}$ on probe-molecule separation. Note that the leading order correction terms in Eq. (4) depend on the force gradient. With three linearly independent measurements based on the current and its harmonics we can model the force interaction $F(Z)$ by using Eq. (4) to get a best fit to the $\phi_{A 1-3}$ data in Fig. 2. It is important to stress that the relationship between $\phi_{A 1}, \phi_{A 2}$, and $\phi_{A 3}$ severely restricts the choice of possible functional forms for $F(Z)$. The green curves in Fig. 2 assume that the

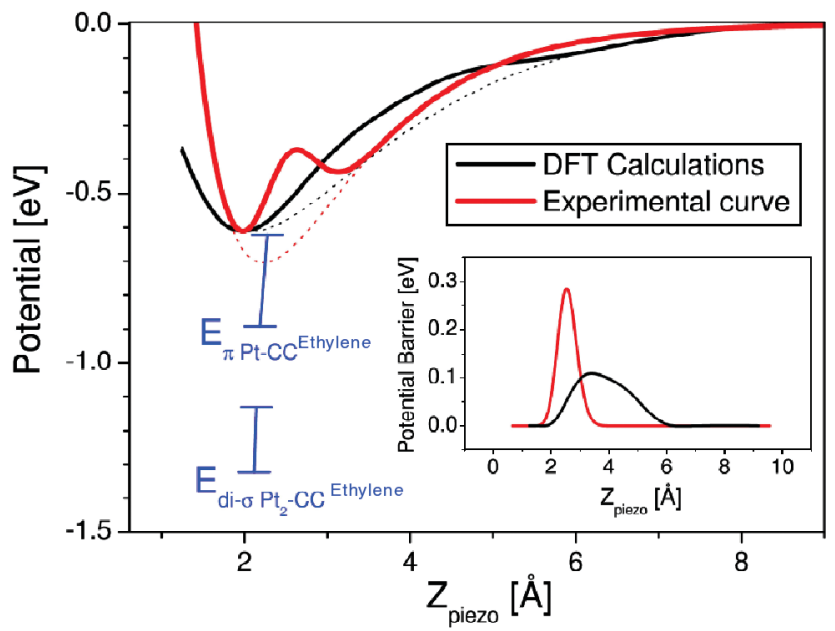

FIG. 4 (color). The probe- $\mathrm{C}=\mathrm{C}$ moiety interaction potential determined using the density functional theory (DFT) calculated vertical displacement of the $\mathrm{C}=\mathrm{C}$ moiety (solid black line) and by fitting the experimental data in Figs. 1(c)-1(e) and 2 using the Morse + barrier potential (solid red line). The dotted lines represent interaction potentials without barrier. The horizontal blue bars connected by lines represent the calculated energy and bondlength ranges for the $\pi$ and di- $\sigma$ bonded ethylene species on a $\mathrm{Pt}$ surface with various numbers of relaxed Pt atoms [20]. The inset shows the result after subtracting the dotted from the solid curves and represents the potential barriers found in the DFT calculations (black curve) and in the experiment (red curve). 
interaction force can be modeled as a negative gradient of a Morse potential between the probe and molecule [10]. The resulting fit is poor but demonstrates that the long-range attractive interaction at large separation causes a small increase in $\phi_{A 1-3}$ whereas the short-range repulsion lead to a dramatic reduction in $\phi_{A 1-3}$ at smaller separations. This behavior is similar to that reported by Chen and Hamers [10].

Guided by these DFT results, we now model the data in Fig. 2 assuming a potential function that includes a Morse term and a repulsive asymmetric-Gaussian barrier centered at some distance from the surface. The resulting fit (solid black curve in Fig. 2) used the same values of the parameters for all three experimental $\phi_{A 1-3}$ curves, and provides an excellent fit to all data. The corresponding force gradient $d F / d Z$ (dotted blue curves) is also plotted in Fig. 2(a) and qualitatively matches $\phi_{A 1}$, reflecting the presence of the $d F / d Z$ term in Eq. (4). The main feature in $\phi_{A 2}$ and $\phi_{A 3}$ is shifted in position and has different amplitude in comparison with $\phi_{A 1}$ and $d F / d Z$ [see Figs. 2(b) and 2(c)] due to the presence of additional terms in Eq. (4).

The Morse+asymmetric-Gaussian potential that results from this analysis is shown as the solid red curve in Fig. 4, while the asymmetric-Gaussian barrier term itself is shown separately in the inset. (Note that the $Z$ scale of the experimental curve has been shifted to align its minimum to the theoretical one, and this shift has been applied to all figures. The $Z$ scale on the Pt surface is based on theory [6]). A comparison of the theoretical and experimental potentials reveals that although both have qualitatively the same features, the calculated barrier is wider and shifted (see the inset in Fig. 4), which likely reflects our limited computational resources, precluding fully relaxed calculations with realistic probes. The experiment shows a weak-binding interaction of $0.6 \mathrm{eV}$, which is consistent with the known strength of $\mathrm{C}=\mathrm{C} \rightarrow \mathrm{Pt}, p \pi \rightarrow d \pi$ complexes [20]. Constraining the computational curve to have the experimental binding energy yields a spring constant of $20 \mathrm{~N} \mathrm{~m}^{-1}$ and a vibrational frequency of about $650 \mathrm{~cm}^{-1}$, which is in the range of the HREELS peaks assigned to the ring motion of 1,3-CHD chemisorbed on Si(111) surface [21]. Together, these data confirm that we are indeed sensing the relaxation of the $\mathrm{C}=\mathrm{C}$ moiety during the approach to contact, and that the potential curve derived from this analysis reflects the probe-molecule interaction. More accurate modelling should include polarization $\left(\phi_{A}\right.$ dependence on $Z$ ) and electric field effects and lead to an even better data fit especially in the small separation region where the probe-molecule bond is formed.

The present method is sensitive to the local relaxation of the molecule and is a powerful technique to evaluate forces during contact formation. For Pt, apart from a weak attractive interaction, the current is well described by Eq. (1), which is in direct contrast with the behavior of 1,3-CHD on $\mathrm{Si}(100)$. The different behavior of these systems reflects differences in bond stiffness. 1,3-CHD molecules relax in the presence of the probe over a range of separations, whereas the strongly bonded $\mathrm{Pt}$ atoms respond only when the probe is close to contact and is quickly followed by neck formation. Relaxation is expected to be important for tethered molecular systems in general so that this method is applicable to molecular contact studies at low biases [where Eq. (1), is valid] and for systems in which the density of states in the tunneling window defined by the bias are effectively constant during the approach trajectory.

This work was supported by Science Foundation Ireland under Grant No. 00/PI.1/C077A.2.

*Present address: NIST, 100 Bureau Drive, Mailstop 8372, Gaithersburg, MD 20899, USA.

†Electronic address: jboland@tcd.ie

[1] G. I. Gittins, D. Bethell, D. J. Schiffrin, and R. J. Nichols, Nature (London) 408, 67 (2000).

[2] A. Nitzan and M. A. Ratner, Science 300, 1384 (2003).

[3] B. Xu and N. Tao, Science 301, 1221 (2003).

[4] K. W. Hipps, Science 294, 536 (2001).

[5] A. R. Champagne, A. N. Pasupathy, and D. C. Ralph, Nano Lett. 5, 305 (2005).

[6] J. B. Pethica and A.P. Sutton, J. Vac. Sci. Technol. A 6, 2490 (1988).

[7] J.R. Smith, G. Bozzolo, A. Banerjea, and J. Ferrante, Phys. Rev. Lett. 63, 1269 (1989).

[8] Y. Sun, H. Mortensen, S. Schär, A. S. Lucier, Y. Miyahara, P. Grütter, and W. Hofer, Phys. Rev. B 71, 193407 (2005).

[9] G. Binnig, N. Garcia, H. Rohrer, J. M. Soler, and F. Flores, Phys. Rev. B 30, 4816 (1984).

[10] C. J. Chen and R. J. Hamers, J. Vac. Sci. Technol. B 9, 503 (1991).

[11] Scanning Tunneling Microscopy and Spectroscopy: Theory, Techniques, and Applications, edited by D. A. Bonnell (VCH, Inc., New York, 1993).

[12] A. Halbritter, Sz. Csonka, G. Milhàly, E. Jurdik, O. Yu. Kolesnychenko, O. I. Shklyarevskii, S. Speller, and H. van Kempen, Phys. Rev. B 68, 035417 (2003).

[13] CRC Handbook of Chemistry and Physics, edited by R. C. Weast, M. J. Astle, and W.H. Beyer (CRC Press, Inc., Boca Raton, Florida, 1987).

[14] K. Hata, S. Yoshida, and H. Shigekawa, Phys. Rev. Lett. 89, 286104 (2002).

[15] L. C. Teague and J. J. Boland, Thin Solid Films 464-465, 1 (2004); J. Phys. Chem. B 107, 3820 (2003).

[16] B. Naydenov, L. C. Teague, P. Ryan, and J. J. Boland, Nano Lett. 6, 1752 (2006).

[17] J. P. Perdew and A. Zunger, Phys. Rev. B 23, 5048 (1981).

[18] A. H. MacDonald, Phys. Rev. B 18, 5897 (1978).

[19] M. D. Segall, P. J. D. Lindan, M. J. Probert, C. J. Pickard, P. J. Hasnip, S. J. Clark, and M. C. Payne, J. Phys. Condens. Matter 14, 2717 (2002).

[20] G. W. Watson, R.P.K. Wells, D. J. Willock, and J. Hutchings, J. Phys. Chem. B 104, 6439 (2000).

[21] F. Tao, Z. H. Wang, and G. Q. Xu, Surf. Sci. 530, 203 (2003). 